\title{
EQUIVARIANT SMOOTHING THEORY
}

\author{
BY R. LASHOF
}

Given a finite group $G$ acting on a topological manifold $M$, when can we put a smooth structure on $M$ such that $G$ acts smoothly? Our approach to this problem is via equivariant immersion theory. This generalizes the immersion theory approach of [12], and we begin by reviewing these ideas. Details will appear in [13].

1. The immersion approach to smoothing theory. A map $\alpha: M_{1}^{n} \rightarrow M_{2}^{n}$ between $n$-dimensional topological manifolds is called a (topological) immersion if $\alpha$ is a local homeomorphism. Of course, a smooth immersion is a topological immersion of the underlying topological manifolds. The basis of the immersion approach to smoothing is the following trivial lemma:

LEMMA 1. A topological immersion $\alpha$ of a topological manifold $M^{n}$ into a smooth manifold $V^{n}$ defines a unique smooth structure on $M$ such that a becomes a smooth immersion.

In fact, define smooth local coordinates on $M$ by pulling back the local coordinates on $V$ via the local homeomorphisms. We will denote this smooth structure by $\boldsymbol{M}_{\alpha}$.

Recall that the differential of a smooth immersion $f: V_{1}^{n} \rightarrow V_{2}^{n}$ induces a bundle homomorphism $d f: T V_{1} \rightarrow T V_{2}$ of the tangent vector bundles which is an isomorphism on fibres. Call such a bundle homomorphism a representation and let $R\left(T V_{1}, T V_{2}\right)$ be the space of representations with the $C^{0}$-topology and $I^{\infty}\left(V_{1}, V_{2}\right)$ the space of smooth immersions with the $C^{\infty}$ topology. The Smale-Hirsch theorem for manifolds of the same dimension states:

THEOREM A (HIRSCH). If no component of $V_{1}$ is closed, $d: I^{\infty}\left(V_{1}, V_{2}\right) \rightarrow$ $R\left(T V_{1}, T V_{2}\right)$ is a weak homotopy equivalence. The relative version for immersions modulo a given immersion on a neighborhood of a closed subset $A$ holds, provided $\overline{M-A}$ has no compact components.

For a topological manifold $M$ we have Milnor's tangent microbundle [15], [12]. Since the fibre of $\tau M$ over $p \in M$ is essentially a neighborhood germ, a local homeomorphism $f: M_{1} \rightarrow M_{2}$ defines a microbundle representation

An invited address delivered to the American Mathematical Society in St. Louis, Missouri, January 27, 1977; received by the editors May 10, 1977.

AMS (MOS) subject classifications (1970). Primary 57E10, 57E15, 57D10; Secondary 57A30, 57A35, 57A55, 55F35.

Key words and phrases. Compact transformation groups, smoothing, topological manifolds, engulfing, immersions, classifying spaces. 
$d f: \tau M_{1} \rightarrow \tau M_{2}$. (Explicitly, the total space of $\tau M$ is any neighborhood $U$ of the diagonal in $M \times M$ and $d f=f \times f \mid U, U$ sufficiently small.) Lees? topological immersion theorem [14] for manifolds of the same dimension states:

THEOREM B. If no component of $M_{1}$ is closed, $d: I^{t}\left(M_{1}, M_{2}\right) \rightarrow$ $R^{t}\left(\tau M_{1}, \tau M_{2}\right)$ is a weak homotopy equivalence .

Here the "space" $I^{t}\left(M_{1}, M_{2}\right)$ of topological immersions must be treated as a simplicial set and similarly for $R^{t}\left(\tau M_{1}, \tau M_{2}\right)$ [12]. Since each $n$-dimensional microbundle contains an essentially unique $R^{n}$ bundle, and these two categories of bundles are equivalent by Kister's theorem [10], we can also consider $R\left(\tau M_{1}, \tau M_{2}\right)$ to be the singular complex of the space of $R^{n}$ bundle representations. Lees' theorem is proved following the scheme of Haefliger and Poenaru [5] for piecewise linear immersions after proving a topological isotopy extension theorem based on the work of Kirby [8].

By taking essentially the smooth singular complex $I^{s}\left(V_{1}, V_{2}\right)$ of $I^{\infty}\left(V_{1}, V_{2}\right)$ and the singular complex $R^{s}\left(T V_{1}, T V_{2}\right)$ of $R\left(T V_{1}, T V_{2}\right)$ we get a homotopy commutative diagram:

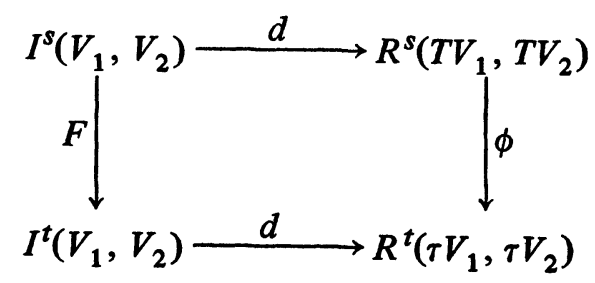

where $F$ is obtained by forgetting the smooth structure and $\phi$ by embedding $T V$ as a neighborhood of the diagonal in $V \times V$ via the exponential map and observing that the topological differential and smooth differential then agree up to a natural homotopy.

As an example, if $\tau M^{n}$ is trivial, i.e., equivalent to $M \times R^{n}$, we can obviously construct a microbundle representation of $\tau M$ into $\tau R^{n}$. By Theorem B, if $M$ is open, there is a topological immersion $\alpha: M \rightarrow R^{n}$, which defines a smooth structure $M_{\alpha}$ on $M$ by Lemma 1 .

More generally (and avoiding technicalities), if $\tau M$ contains a vector bundle $\xi$ and $U$ is a contractible open set of $M, \xi \mid U$ is trivial and we have a vector bundle representation $\xi \mid U \rightarrow T R^{n}$ and hence a microbundle representation $\tau U=\tau M \mid U \rightarrow \tau R^{n}$, which induces a smoothing of $U$. Further, because the smoothing of $U$ corresponds to the trivialization of $\xi \mid U$, if $U^{\prime}$ is another such neighborhood, the smoothing of $U \cap U^{\prime}$ can be extended to a smoothing of $U^{\prime}$ corresponding to $\xi \mid U^{\prime}$. That is, by Theorem A (relative version), there is a smooth immersion $f$ of $U \cap U^{\prime}$ in $R^{n}$ whose differential extends to a vector bundle representation of $\xi \mid U^{\prime} \rightarrow T R^{n}$. By Theorem B (relative version), $f$ extends to a topological immersion $f^{\prime}: U^{\prime} \rightarrow R^{n}$ which induces a smooth structure on $U^{\prime}$ extending that on $U \cap U^{\prime}$. Thus by induction over a countable open cover we get a smoothing of $M$ corresponding to the reduction $\xi$ of $\tau M$, provided $M$ is open.

Define two smooth structures $M_{\alpha}, M_{\beta}$ on a topological manifold $M$ to be 
isotopic if $\mathrm{id}_{M}$ is ambient isotopic as a homeomorphism of $M_{\alpha}$ onto $M_{\beta}$ to a diffeomorphism. Then in [12] (see also [9]), we prove for general (in particular, closed) $M$ :

THEOREM C. If $n \neq 4$, the isotopy classes of smoothings of $M^{n}$ are in bijective correspondence with the homotopy classes of reductions of $\tau M$ to a vector bundle.

The condition $n \neq 4$ comes from the fact that the immersion theorem does not apply to closed manifolds so that we have to apply it to $M-p$. In order to extend the smoothing over $p$, and to prove uniqueness up to isotopy, the smoothing near $p$ has to be "straightened out" and this requires engulfing techniques which hold for $n \geqslant 5$. The case $n \leqslant 3$ is classical.

Now homotopy classes of reductions of $\tau M$ correspond to homotopy classes of lifts of the classifying map $\tau: M \rightarrow B$ Top ${ }_{n}$ of the tangent $R^{n}$ bundle to $B O_{n}$. Here Top ${ }_{n}$ is the group of homeomorphisms of $R^{n}$ with the $C^{0}$-topology and $O_{n}$ is the orthogonal group. The map of classifying spaces $B O_{n} \rightarrow B$ Top $_{n}$ may be considered as a fibre space with fibre Top $n / O_{n}$. Thus the obstructions to smoothing and uniqueness lie in $\pi_{i}\left(\operatorname{Top}_{n} / O_{n}\right), i \leqslant n$.

The analogue of the fact that $O_{n+1} / O_{n}=S^{n}$ is the result [11] that Top $_{n+1} /$ Top $_{n}=S^{n} \times B C\left(S^{n}\right)$. The group $C\left(S^{n}\right)$ is the pseudoisotopy or concordance group of $S^{n}$; i.e., homeomorphisms of $I \times S^{n}, I=[0,1]$, which are the identity on $0 \times S^{n}$. Thus we have a homotopy theoretic fibration Top $_{n} / O_{n} \rightarrow$ Top $_{n+1} / O_{n+1}$ with fibre $C\left(S^{n}\right)$. For $n \leqslant 3$ every manifold has a unique smoothing up to isotopy. For $n \geqslant 5$, it can be shown that $\pi_{i} C\left(S^{n}\right)=$ 0 for $i \leqslant n+1$. In fact, by surgery arguments of [7] and [16], $\pi_{i} C\left(S^{n}\right)=$ $\pi_{i} C^{p l}\left(S^{n}\right)$, the piecewise linear group. The result then follows from Haefliger and Wall's analysis of $\pi_{i} P L_{n+1} / P L_{n}$, see [6]. Hence

$$
\pi_{i}\left(\operatorname{Top}_{n} / O_{n}\right)=\pi_{i}(\mathrm{Top} / O), \quad i \leqslant n+1,
$$

where

$$
\text { Top }=\underset{n \rightarrow \infty}{\operatorname{ind} \operatorname{Lim}} \operatorname{Top}_{n} \text { and } 0=\operatorname{Lim} O_{n}
$$

under inclusion. Finally, the computation of $\pi_{i}$ Top $/ O$ can be reduced to computing homotopy groups of spheres by surgery methods. In principle, therefore, one can compute the obstruction groups.

2. Equivariant smoothing. Let $G$ be a finite group. A topological or smooth $G$-immersion of $G$-manifolds is just an immersion which is a $G$-map. The equivariant version of Lemma 1 is:

LEMMA 1 EQ. $A$ topological $G$-immersion $\alpha$ of a topological G-manifold $M^{n}$ into a smooth $G$-manifold $V^{n}$ defines a unique equivariant smooth structure $M_{\alpha}$ on $M$ such that $\alpha$ becomes an equivariant smooth immersion.

If $V$ is a smooth $G$-manifold, the differential of the action of $G$ on $V$ induces an action of $G$ on $T V$ making it into a $G$-vector bundle [3] and [17]:

Definition. A $G$-vector bundle is a vector bundle $p: E \rightarrow B$ where $E$ and $B$ are $G$-spaces, $p$ is a $G$-map, and the action of $G$ on $E$ is through vector bundle maps.

The differential of a smooth $G$-immersion $f: V_{1}^{n} \rightarrow V_{2}^{n}$ induces a $G$-bundle 
homomorphism df: $T V_{1} \rightarrow T V_{2}$ which is an isomorphism of fibres. Let $R_{G}\left(T V_{1}, T V_{2}\right)$ be the space of $G$-vector bundle representations and $I_{G}^{\infty}\left(V_{1}, V_{2}\right)$ the space of $G$-immersions. Bierstone [3] has given an equivariant Gromov theory proving in particular a $G$-version of Theorem A. To state it we first need the definitions:

Definition (BREDON [4]). A topological $G$-manifold $M$ is called locally smooth if $M$ has an atlas of $G$-invariant open sets $U$, such that each $U$ admits an equivariant smoothing.

Definition. Let $M_{(H)}$ be the union of orbits of type $(H) . M_{(H)}$ is $G$ invariant and a bundle over $M_{(H)} / G$ with fibre $G / H$ [4]. If $M$ is a (locally) smooth $G$-manifold, $M_{(H)}$ is a (locally) smooth submanifold. We say $M$ satisfies the Bierstone Condition if no $G$-component of $M_{(H)}$ is a closed manifold. (A $G$-component of $M_{(H)}$ is the preimage of a component of $M_{(H)} / G$.)

THEOREM A EQ. (BIERSTONe [3]). If $V_{1}, V_{2}$ are smooth G-manifolds of the same dimension and $V_{1}$ satisfies the Bierstone Condition, $d: I_{G}^{\infty}\left(V_{1}, V_{2}\right) \rightarrow$ $R_{G}\left(T V_{1}, T V_{2}\right)$ is a weak homotopy equivalence.

Again this theorem has a semisimplicial version. By methods analogous to the $G$-trivial case we get a $G$-version of Theorem B.

THEOREM B EQ. If $M_{1}, M_{2}$ are locally smooth G-manifolds of the same dimension and $M_{1}$ satisfies the Bierstone Condition, $d: I_{G}^{t}\left(M_{1}, M_{2}\right) \rightarrow$ $R_{G}^{t}\left(\tau M_{1}, \tau M_{2}\right)$ is a weak homotopy equivalence.

Again $I_{G}^{t}\left(M_{1}, M_{2}\right)$ and $R_{G}^{t}\left(\tau M_{1}, \tau M_{2}\right)$ are simplicial sets. Also $\tau M$ is a $G$-microbundle; i.e., $G$ acts on the total space through microbundle maps.

The notion of local triviality for $G$-vector bundles is somewhat more involved than for ordinary vector bundles: If $\xi$ is a $G$-vector bundle over a completely regular $G$-space $X$, for each $x \in X$ there is a slice $S_{x}$ (i.e., the orbit $G x$ through $x$ has a $G$-neighborhood $G S_{x}, G$-equivalent to $G \times_{G_{x}} S_{x}$ ), such that $\xi \mid G S_{x}$ is equivalent to the $G$-vector bundle $1_{\rho}\left(S_{x}\right): G \times{ }_{G_{x}}\left(S_{x} \times R_{\rho}^{n}\right) \rightarrow$ $G \times{ }_{G_{x}} S_{x}$ (obvious projection), where $R_{\rho}^{n}$ is an orthogonal $G_{x}$ space, $\rho: G_{x} \rightarrow$ $O_{n}$ a representation.

Note that since $M$ is locally smooth $\tau M$ is locally $G$-equivalent to a $G$-vector bundle and hence locally $G$-trivial in the above sense. One may prove a $G$-Kister theorem for locally $G$-trivial microbundles and show the category of locally $G$-trivial microbundles coincides with the category of locally $G$-trivial $G-R^{n}$ bundles.

Now $T\left(G \times{ }_{G_{x}} R_{\rho}^{n}\right)=G \times{ }_{G_{x}}\left(R_{\rho}^{n} \times R_{\rho}^{n}\right)$ and we have an obvious $G$ vector bundle map of $1_{\rho}\left(S_{x}\right) \rightarrow T\left(G \times{ }_{G_{x}} R_{\rho}^{n}\right)$ sending $S_{x}$ to $0 \in R_{\rho}^{n}$.

Thus again we have that if $\tau M$ contains a $G$-vector bundle $\xi$ we can cover $M$ by $G$-invariant neighborhoods $U=G S_{x}$ such that $\xi \mid U$ is $G$-trivial and hence we get a $G$-immersion $U \rightarrow G \times \times_{G_{x}} R_{\rho}^{n}$ and a $G$-smoothing of $U$ by Lemma 1 eq. Then using Theorems $A$ eq. and $B$ eq., we get by an argument completely analogous to the $G$-trivial case that if $M$ satisfies the Bierstone Condition and $\tau M$ reduces to a $G$-vector bundle $\xi$, then $M$ has a $G$-smoothing corresponding to the reduction of $\tau M$ to $\xi$ (cf. [2]).

To obtain a result for arbitrary $G$-manifolds we must use a $G$-engulfing 
theorem. This is proved from the ordinary engulfing theorem by inducing up the orbit types and leads to:

THEOREM C EQ. If $\operatorname{dim} H \neq 4$ for any $H \subset G$, the isotopy classes of $G$-smoothings of $M$ are in bijective correspondence with the homotopy classes of $G$-vector bundle reductions of $\tau M$.

We remark that it isn't necessary to assume $M$ is locally smooth, because it is easy to see that if $\tau M$ reduces to a $G$-vector bundle then $M$ must be locally smooth.

The obstructions to reducing $\tau M$ to a $G$-vector bundle lies in $\pi_{i}\left(\operatorname{Top}_{n}^{\rho} / O_{n}^{\rho}\right)$, where $\rho: H \rightarrow O_{n}$ and $\operatorname{Top}_{n}^{\rho}\left(O_{n}^{\rho}\right)$ is the subgroup of $\operatorname{Top}_{n}\left(O_{n}\right)$ commuting with the orthogonal action of $H$.

Now $R_{\rho}^{n}=R_{\alpha}^{k} \oplus R^{l}, k+l=n$, where we have split off the trivial representations. Write Top ${ }_{n}^{\rho}=\operatorname{Top}_{k+l}^{\alpha}$ and $O_{n}^{\rho}=O_{k+l}^{\alpha}$. Then if we let $C^{\alpha}\left(S^{k+l}\right)$ be the subgroup of $C\left(S^{k+l}\right)$ commuting with the action of $H$ on $I \times S^{k+l}$ (trivial action on $I$, orthogonal action on $S^{k+l}$ ), we again have a fibration:

$$
C^{\alpha}\left(S^{k+l}\right) \rightarrow \text { Top }_{k+l}^{\alpha} / O_{k+l}^{\alpha} \rightarrow \operatorname{Top}_{k+l+1}^{\alpha} / O_{k+l+1}^{\alpha} .
$$

Here however, the groups $\pi_{i} C^{\alpha}\left(S^{k+l}\right)$ are not zero in general. In principle, they can be computed by methods of Anderson and Hsiang [1]. In particular, if $H$ acts freely on $S^{h-1}$ via $\alpha$ then $\pi_{i} C^{\alpha}\left(S^{h+l}\right) \simeq \pi_{i} C^{\alpha}\left(S^{h+l} \bmod S^{l}\right) \oplus$ $\pi_{i} C\left(S^{l}\right)$; and if $k+l \geqslant 6$, Anderson and Hsiang have shown:

$$
\pi_{i} C\left(S^{h+l} \bmod S^{l}\right) \simeq \begin{aligned}
& K_{-l+1+i}(Z(H)), \quad i<l-1 \\
& \tilde{K}_{0}(Z(H)), \quad i=l-1 \\
& \mathrm{Wh}_{1}(H), \quad i=l \\
& \pi_{i-l-1} C\left(L \times D^{l+1}\right), \quad i>l
\end{aligned}
$$

where $L=S^{h-1} / H$ and the $K_{-j}$ are Bass' algebraic $K$ groups.

Let $M^{n}$ be a locally smooth $H$-manifold for which the action is semifree. Suppose $\operatorname{dim} M^{H}=l, n=k+l$ and $\alpha: H \rightarrow O_{k}$ is the representation of $H$ on the normal disc to $M^{H}$. Then the obstructions to $H$-smoothing lie in Top $_{k+l}^{\alpha} / O_{k+l}^{\alpha}$ and in $\operatorname{Top}_{n} / O_{n}$ if $\operatorname{dim} M^{H} \neq 4$ and $\operatorname{dim} M \neq 4$. For this we need know $\pi_{i}\left(\operatorname{Top}_{k+l}^{\alpha} / O_{k+l}^{\alpha}\right)$ only for $i<l$ and $\pi_{i} \operatorname{Top}_{n} / O_{n}$ for $i \leqslant n$.

Now Top $l / O_{l}$ is a retract of $\operatorname{Top}_{k+l}^{\alpha} / O_{k+l}^{\alpha}$. We also have the inclusion of $A^{\alpha}\left(S^{k-1}\right) / O_{k}^{\alpha} \rightarrow$ Top $_{k+1}^{\alpha} / O_{k+1}^{\alpha}$, where $A^{\alpha}\left(S^{k-1}\right)=$ group of homeomorphisms of $S^{k-1}$ commuting with $\alpha$. It can be shown that this map induces a split injection

$$
\pi_{i} \tilde{A}^{\alpha}\left(S^{k-1}\right) / O_{k}^{\alpha} \rightarrow \pi_{i}\left(\operatorname{Top}_{k+l}^{\alpha} / O_{k+l}^{\alpha}, \operatorname{Top}_{l} / O_{l}\right), \quad i \leqslant l ;
$$

where $\tilde{A}^{\alpha}\left(S^{k-1}\right)=$ group of block homeomorphisms of $S^{k-1}$ commuting with $\alpha$ (see [12]). Hence we get a split injection:

$$
\pi_{i}\left(\tilde{A}^{\alpha}\left(S^{k-1}\right) / O_{k}^{\alpha}\right) \oplus \pi_{i}\left(\operatorname{Top}_{l} / O_{l}\right) \rightarrow \pi_{i} \operatorname{Top}_{k+l}^{\alpha} / O_{k+l}^{\alpha}, \quad i \leqslant l .
$$

Further, from the fibration above, using the fact that $\pi_{i} C\left(S^{l}\right)=0, i<l+1$, we get the exact sequence: 


$$
\begin{aligned}
0 & \rightarrow \pi_{l+1}\left(\tilde{A}^{\alpha}\left(S^{k-1}\right) / O_{k}^{\alpha}\right) \oplus \pi_{l+1}\left(\operatorname{Top}_{l+1} / O_{l+1}\right) \\
& \rightarrow \pi_{l+1}\left(\operatorname{Top}_{k+l+1}^{\alpha} / O_{k+l+1}^{\alpha}\right) \rightarrow \mathrm{Wh}_{1}(H) \rightarrow \pi_{l}\left(\operatorname{Top}_{k+l}^{\alpha} / O_{k+l}^{\alpha}\right) \\
& \rightarrow \pi\left(\operatorname{Top}_{k+l+1}^{\alpha} / O_{k+l+1}^{\alpha}\right) \rightarrow \tilde{K}_{0}(Z(H)) \rightarrow \pi_{l-1}\left(\operatorname{Top}_{k+l}^{\alpha} / O_{k+l}^{\alpha}\right) \\
& \rightarrow \pi_{l-1}\left(\operatorname{Top}_{k+l+1}^{\alpha} / O_{k+l+1}^{\alpha}\right) \rightarrow K_{-l+1}(Z(H)) \rightarrow \pi_{0}\left(\operatorname{Top}_{k+l}^{\alpha} / O_{k+l}^{\alpha}\right) \\
& \rightarrow \pi_{0}\left(\operatorname{Top}_{k+l+1}^{\alpha} / O_{k+l+1}^{\alpha}\right) \rightarrow K_{-l}(Z(H)) .
\end{aligned}
$$

Of course, $\pi_{l+1}\left(\operatorname{Top}_{l+1} / O_{l+1}\right) \simeq \pi_{l+1}(\operatorname{Top} / O)$. Also $\pi_{i}\left(\tilde{A}^{\alpha}\left(S^{k-1}\right) / O_{k}^{\alpha}\right)$ can be computed up to extension from the surgery exact sequence for $L$.

Finally, we note the following results of Bass and others for the algebraic $\boldsymbol{K}$-groups.

For $\pi$ abelian, $K_{-j}(Z(\pi))=0$ for $j>1$.

For $\pi$ abelian and prime power order, $K_{-1}(Z(\pi))=0$.

For $\pi$ cyclic of order $p, K_{0}(Z(\pi))=$ class group of $Q\left(e^{2 \pi i / p}\right)$.

For $\pi$ finite $\tilde{K}_{0}(Z(H))$ is finite.

\section{REFERENCES}

1. D. Anderson and W. C. Hsiang, The functors $K_{-i}$ and pseudo-isotopies of polyhedra, Ann. of Math. (2) 105 (1977), 201-224.

2. G. Anderson, Classification of structures on abstract manifolds, Mass. Inst. of Tech., Cambridge, 1976 (preprint).

3. E. Bierstone, Equivariant Gravov theory, Topology 13 (1974), 327-346.

4. G. Bredon, Introduction to compact transformation groups, Academic Press, New York, 1972.

5. A. Haefliger and V. Poenaru, La classification des immersions combinatoires, Inst. Hautes Études Sci. Publ. Math. 23 (1964), 75-91.

6. A. Haefliger and C. T. C. Wall, Piecewise linear bundles in the stable range, Topology 4 (1965), 209-214.

7. W. C. Hsiang and J. Shaneson, Fake tori, the annulus conjecture, and the conjecture of Kirby, Proc. Nat. Acad. Sci. USA 62 (1969), 687-691.

8. R. Kirby, Stable homeomorphisms and the annulus conjecture, Ann. of Math. (2) 89 (1969), 575-582.

9. R. Kirby and L. Siebenmann, Essays on topological manifolds, smoothings and triangulations (to appear).

10. J. Kister, Microbundles are fibre bundles, Ann. of Math. (2) 80 (1964), 190-199.

11. N. Kuiper and R. Lashof, Microbundles and bundles. I, Invent. Math. 1 (1968), 1-17.

12. R. Lashof, The immersion approach to triangulation and smoothing, (AMS Summer Inst., Madison, Wisc., 1970) Proc. Sympos. in Pure Math., vol. 22, Amer. Math. Soc., Providence, R.I., 1971, pp. 131-164.

13. R. Lashof and M. Rothenberg, G-smoothing theory, (Proc. AMS Summer Inst., Stanford, 1976) (to appear).

14. J. Lees, Immersions and surgeries on topological manifolds, Bull. Amer. Math. Soc. 75 (1969), 529-534.

15. J. Milnor, Microbundles, Proc. Internat. Congress Math. (Stockholm, 1962), Inst. MittagLeffler, Djorsholm, 1963.

16. C. T. C. Wall, On homotopy tori and the annulus theorem, Bull. London Math. Soc. 1 (1969), 95-97.

17. A. Wasserman, Equivariant differential topology, Topology 8 (1969), 127-150.

Department of Mathematics, University of Chicago, Chicago, Illinois 60637 